\title{
Erratum: Detecting gravitational waves from precessing binaries of spinning compact objects: Adiabatic limit \\ [Phys. Rev. D 67, 104025 (2003)]
}

Alessandra Buonanno, Yanbei Chen, and Michele Vallisneri

(Received 11 July 2006; published 31 July 2006)

DOI: 10.1103/PhysRevD.74.029904

PACS numbers: 04.30.Db, 04.25.Nx, 04.80.Nn, 95.55.Ym, 99.10.Cd

A small error in the computation of the gravitational wave flux in Refs. [1,2] propagates to the calculations of Ref. [3], and consequently to Eq. (1) of this paper, which should be modified to read

$$
\begin{aligned}
\frac{\dot{\omega}}{\omega^{2}}= & \frac{96}{5} \eta(M \omega)^{5 / 3}\left\{1-\frac{743+924 \eta}{336}(M \omega)^{2 / 3}-\left(\frac{1}{12} \sum_{i=1,2}\left[\chi_{i}\left(\hat{\mathbf{L}}_{N} \cdot \hat{\mathbf{S}}_{i}\right)\left(113 \frac{m_{i}^{2}}{M^{2}}+75 \eta\right)\right]-4 \pi\right)(M \omega)\right. \\
& +\left(\frac{34103}{18144}+\frac{13661}{2016} \eta+\frac{59}{18} \eta^{2}\right)(M \omega)^{4 / 3}-\frac{1}{48} \eta \chi_{1} \chi_{2}\left[247\left(\hat{\mathbf{S}}_{1} \cdot \hat{\mathbf{S}}_{2}\right)-721\left(\hat{\mathbf{L}}_{N} \cdot \hat{\mathbf{S}}_{1}\right)\left(\hat{\mathbf{L}}_{N} \cdot \hat{\mathbf{S}}_{2}\right)\right](M \omega)^{4 / 3} \\
& -\frac{1}{672}(4159+15876 \eta) \pi(M \omega)^{5 / 3}+\left[\left(\frac{16447322263}{139708800}-\frac{1712}{105} \gamma_{E}+\frac{16}{3} \pi^{2}\right)+\left(-\frac{273811877}{1088640}+\frac{451}{48} \pi^{2}-\frac{88}{3} \hat{\theta}\right) \eta\right. \\
& \left.\left.+\frac{541}{896} \eta^{2}-\frac{5605}{2592} \eta^{3}-\frac{856}{105} \log \left[16(M \omega)^{2 / 3}\right]\right](M \omega)^{2}+\left(-\frac{4415}{4032}+\frac{358675}{6048} \eta+\frac{91495}{1512} \eta^{2}\right) \pi(M \omega)^{7 / 3}\right\} .
\end{aligned}
$$

The changes affect the $2 \mathrm{PN}$ and $3 \mathrm{PN}$ coefficients that multiply $(M \omega)^{5 / 3}$ and $(M \omega)^{7 / 3}$, respectively. By means of order-ofmagnitude arguments and of limited numerical tests, we have determined that the gravitational waveform corrections resulting from these changes are too small to affect the main quantitative results of this paper.

In addition, we point out that the previously unknown value of the parameter $\hat{\theta}$, which enters the gravitational wave flux at the 3PN order, has been determined to be 1039/4620 [4].

Last, all the spins $\left(\mathbf{S}_{1}, \mathbf{S}_{2}\right.$, and $\left.\mathbf{S}_{\text {eff }}\right)$ that appear in Eq. (11) should be divided by $M^{2}$.

[1] L. Blanchet, Phys. Rev. D 54, 1417 (1996); Classical Quantum Gravity 15, 113 (1998).

[2] L. Blanchet, G. Faye, B. R. Iyer, and B. Joguet, Phys. Rev. D 65, 061501(R) (2002); L. Blanchet, B. R. Iyer, and B. Joguet, Phys. Rev. D 71, 129903(E) (2005).

[3] A. Buonanno, Y. Chen, and M. Vallisneri, Phys. Rev. D 67, 024016 (2003).

[4] L. Blanchet, T. Damour, G. Esposito-Farèse, and B. R. Iyer, Phys. Rev. Lett. 93, 091101 (2004). 